\title{
An Algorithm for Solving a Polynomic Congruence, and its Application to Error-Correcting Codes
}

\author{
By M. H. McAndrew
}

1. Introduction. The solution of $f(x)=0$ in the $p$-adic field may be calculated by the Newton-Raphson process, the iteration of the transformation: $x \rightarrow x-$ $f(x) / f^{\prime}(x)$; as in the real field the formula cannot be applied successfully unless we have an initial approximation sufficiently close to a root for the subsequent iteration to converge. (In the $p$-adic field, "sufficiently close" is equivalent to "congruent to a sufficiently high power of $p$. .") In this paper we deduce a simple criterion to ensure that the initial approximation is suitable and we develop a procedure for calculating the roots of $f(x) \equiv 0\left(\bmod p^{k}\right)$ for any value of $k$, using the above process where applicable and a single-stepping procedure elsewhere. In $\$ 6$ we apply this algorithm to investigate solutions of a congruence connected with the existence of close-packed 'error-correcting binary codes. We deduce that for $n<2^{70}$ and $2 \leqq r \leqq 20$ there are no such codes other than the trivial codes and the Golay code. This result complements results of Shapiro and Slotnick [5] and Selfridge [4] which show that there are no codes for $r=2$, or $r$ an odd integer less than 135, or $n<10^{8}$.

2. Notation. $p$ is a prime and $f(x)$ a polynomial with integer coefficients; $f^{\prime}(x)$ is the formal derivative of $f(x)$. We use the notation $p^{a} \| B$ for " $p$ " $\mid B$ and $p^{a+1} \nmid B$." Define $l(x)$ by $p^{l} \| f^{\prime}(x)$. Define

$$
b(m, x)=\operatorname{Max}\left\{\left[\frac{m+1}{2}\right], m-l(x)\right\} .
$$

We write $l, l_{1}, l_{2}, \cdots$ for $l(x), l\left(x_{1}\right), l\left(x_{2}\right), \cdots$; similarly, for $b, b_{1}, b_{2}, \cdots$ where the relevant value of $m$ is clear from the context. We say $x$ is a solution of type $\mathrm{A} \bmod p^{m}$ if

$$
f(x) \equiv 0\left(\bmod p^{m}\right)
$$

and $m \geqq 2 l+1$. We say $x$ is a solution of type $\mathrm{B} \bmod p^{m}$ if $(1)$ holds and $m \leqq 2 l$.

\section{Properties of Solution-Sets.}

LemmA 1. (i) If $x$ is a solution of type $\mathrm{A} \bmod p^{m}$, then $b=m-l$ and $2 b \geqq m+$ $1 \geqq 2 l+2$.

(ii) If $x$ is a solution of type $\mathrm{B}$ mod $p^{m}$ then $b=[(m+1) / 2]$ and $b \leqq l$.

Proof. These results follow directly from the definition of solution type.

LEMMA 2. If $f(x) \equiv 0\left(\bmod p^{m}\right)$ and $x_{1} \equiv x\left(\bmod p^{b}\right)$, then

(i) $x_{1}$ is a solution mod $p^{m}$ of the same type as $x$.

(ii) $b_{1}=b$.

(iii) If $x$ is of type $\mathrm{A} \bmod p^{m}$ then $l_{1}=l$.

Proof. By hypothesis, $x_{1}=x+u p^{b}$ for integral $u$; hence,

$$
f\left(x_{1}\right)=f(x)+u p^{b} f^{\prime}(x)+v p^{2 b},
$$

Received September 30, 1963. Revised June 24, 1964. 


$$
f^{\prime}\left(x_{1}\right)=f^{\prime}\left(x_{1}\right)+w p^{b},
$$

for integral $v$ and $w$, by Taylor's theorem for polynomials. Now $p^{m} \mid f(x)$ and, by definition of $b, b+l \geqq m$ and $2 b \geqq m$; hence in (2)

$$
f\left(x_{1}\right) \equiv 0\left(\bmod p^{m}\right) .
$$

To complete the proof we distinguish two cases.

(a) If $x$ is a solution of type A mod $p^{m}$ then, by Lemma 1 (i), $b \geqq l+1$; hence, in (3), $p^{l} \| f^{\prime}\left(x_{1}\right)$, i.e., $l_{1}=l$. Therefore $2 l_{1}+1=2 l+1 \leqq m, x_{1}$ is a solution of type A $\bmod p^{m}$, and $b_{1}=m-l_{1}=m-l=b$.

(b) If $x$ is a solution of type $\mathrm{B} \bmod p^{m}$ then, by Lemma 1 (ii), $b \leqq l$; hence, in (3), $l_{1} \geqq b=[(m+1) / 2]$, i.e., $2 l_{1} \geqq m$. Hence $x_{1}$ is a solution of type $\mathrm{B} \bmod p^{m}$ and $b_{1}=[(m+1) / 2]=b$, by Lemma 1 (ii).

This concludes the proof of Lemma 2 .

In view of Lemma 2 , we define a solution-set $\bmod p^{m}$ as the set of all $x_{1}$ with $x_{1} \equiv x\left(\bmod p^{b}\right)$, where $x$ is a solution of $(1)$ and $b=b(m, x)$. We use the notation $(x, b, m)$ for such a solution-set and say $x$ is a representative of it. By Lemma 2 (ii), the value of $b$ is independert of the choice of representative and, by Lemma 2 (i), we may define unambiguously the type of a solution-set as the type of any representative. Let $S(m)$ be the totality of solution-sets $\bmod p^{m}$.

We define an extension to $\bmod p^{m+r}$ of the solution-set $(x, b, m)$ as a solution-set $\left(x_{1}, b_{1}, m+r\right)$ with $x_{1} \equiv x\left(\bmod p^{b}\right)$. Clearly $S(m+r)$ consists of just all extensions to $\bmod p^{m+r}$ of the solution-sets of $S(m)$.

Theorem 1. (i) If $(x, b, m)$ is a solution-set of type $\mathrm{A}$, then it has a unique extension, $\left(x_{1}, b_{1}, m+1\right)$ to $\bmod p^{m+1}$; this extension is also of type $\mathrm{A}$ with $l_{1}=l$ and $b_{1}=b+1$.

(ii) If $(x, b, m)$ is a solution-set of type $\mathrm{B}$, then (a) if $m$ is odd either $(x, b$, $m+1)$ is the unique extension of $(x, b, m)$ to $\bmod p^{m+1}$ or there is no extension to $\bmod p^{m+1}$; (b) if $m$ is even, the extensions to $\bmod p^{m+1}$ are just those $\left(x+s p^{b}\right.$, $b+1, m+1)$ for which $0 \leqq s<p$ and $f\left(x+s p^{b}\right) \equiv 0\left(\bmod p^{m+1}\right)$.

Proof. For any integ ral $s$,

$$
f\left(x+s p^{b}\right)=f(x)+s p^{b} f^{\prime}(x)+v p^{2 b},
$$

for integral $v$.

(i) If $x$ is a solution of type A then, by Lemma 1 (i), $b=m-l$ and $2 b \geqq m+1$; hence, from $(5), f\left(x+s p^{b}\right) \equiv 0\left(\bmod p^{m+1}\right)$ if and only if

$$
p^{-m} f(x)+s p^{-l} f^{\prime}(x) \equiv 0(\bmod p) .
$$

Since $p \nmid p^{-l} f^{\prime}(x),(6)$ has a unique solution $\bmod p$ for $s, s_{0}$ say. Let $x_{1}=x+s_{0} p^{b}$; then the unique extension of $(x, b, m)$ to $\bmod p^{m+1}$ is clearly $\left(x_{1}, b_{1}, m+1\right)$. Further, $l_{1}=l$, by Lemma 2 (iii); hence $m+1>2 l_{1}+1$ and so $\left(x_{1}, b_{1}, m+1\right)$ is of type A with $b_{1}=m+1-l_{1}=m+1-l=b+1$.

(ii) In this case, by Lemma 1 (ii), $b=[(m+1) / 2]$. (a) If $m$ is odd, then $b=(m+1) / 2$; hence $b+l=(m+1) / 2+l \geqq(m+1) / 2+m / 2>m$. Therefore in $(5) f\left(x+s p^{b}\right) \equiv f(x)\left(\bmod p^{m+1}\right)$. Hence if $f(x) \not \equiv 0\left(\bmod p^{m+1}\right)$, then $(x, b, m)$ has no extension to $\bmod p^{m+1}$; if $f(x) \equiv 0\left(\bmod p^{m+1}\right)$ then, since $m+1 \leqq 2 l, x$ is a solution of type $\mathrm{B} \bmod p^{m+1}$ with 


$$
\begin{aligned}
b(m+1, x) & =\left[\frac{m+1+1}{2}\right], \quad \text { by Lemma } 1 \text { (ii) } \\
& =\frac{m+1}{2}, \quad \text { since } m \text { is odd } \\
& =b(m, x),
\end{aligned}
$$

i.e., in this case $(x, b, m+1)$ is the unique extension. (b) If $m$ is even, then $b=m / 2$. For any $s, x+s p^{b}$ is a solution of type $\mathrm{B} \bmod p^{m}$, by Lemma 2 (i), i.e., $l^{\prime}=l\left(x+s p^{b}\right) \geqq m / 2$. If $f\left(x+s p^{b}\right) \equiv 0\left(\bmod p^{m+1}\right)$ then

$$
\begin{aligned}
b\left(m+1, x+s p^{b}\right) & =\operatorname{Max}\left(\left[\frac{m+1+1}{2}\right], m+1-l^{\prime}\right) \\
& =\operatorname{Max}\left(\frac{m+2}{2}, m+1-l^{\prime}\right) \\
& =\frac{m+2}{2}, \quad \text { since } l^{\prime} \geqq \frac{m}{2}, \\
& =b+1 .
\end{aligned}
$$

I.e., the solution-set $\bmod p^{m+1}$ containing $x+s p^{b}$ is just $\left(x+s p^{b}, b+1, m+1\right)$. This completes the proof of Theorem 1 .

Theorem 2. If $(x, b, m)$ is a solution-set of type $\mathrm{A}$ then

$$
f(x)+u f^{\prime}(x) \equiv 0\left(\bmod p^{2 m-2 l}\right)
$$

has a solution $u$, unique mod $p^{m-2 l}$, and $(x+u, 2 m-3 l, 2 m-2 l)$ is the unique extension to $\bmod p^{2 m-2 l}$ of $(x, b, m)$.

Proof. Since $(x, b, m)$ is a solution-set of type A, $m>2 l$. Hence, since $p^{m} \mid f(x)$ and $p^{l} \| f^{\prime}(x)$, equation (7) has a solution for $u$, unique mod $p^{2 m-3 l}$. Further $p^{m-l} \mid u$ since, from $(7), u f^{\prime}(x) \equiv 0\left(\bmod p^{m}\right)$. By Taylor's theorem,

$$
\begin{aligned}
f(x+u) & \equiv f(x)+u f^{\prime}(x)\left(\bmod p^{2 m-2 l}\right) \\
& \equiv 0\left(\bmod p^{2 m-2 l}\right), \quad \text { by }(7) .
\end{aligned}
$$

Therefore $x+u$ is a solution $\bmod p^{2 m-2 l}$ and, since $p^{b}=p^{m-l} \mid u, x+u \in(x, b, m)$. By Theorem 1 (i) the solution-set $(x, b, m)$ has a unique extension $\left(x_{1}, b+1, m+1\right)$ to $\bmod p^{m+1}$, also of type $\mathrm{A}$; by induction it has a unique extension $\left(x_{m-2 l}, b+m-2 l\right.$, $2 m-2 l)$ to $\bmod 2 m-2 l$. Since $x+u$ is a solution $\bmod p^{2 m-2 l}$ this concludes the proof of the theorem.

4. Description of the Algorithm. The solution-sets of an integral polynomial $f(x) \bmod p^{m}$ form a tree with extension as the connective. For example, the solution-sets of $f(x)=(x+1)\left(x^{2}-x+6\right)\left(\bmod 2^{m}\right)$ are depicted in Figure 1. We can construct all the solution-sets by starting with the unique solution-set $\bmod p^{0}$, namely, $(0,0,0)$, and calculate the solution-sets $\bmod p^{m+1}$ as the extensions of the solution-sets $\bmod p^{m}$. For a solution-set of type A we may construct its extension to $\bmod p^{N}$ in about $\log _{2} N$ steps by the algorithm of Theorem 2 . For solution-sets of type $\mathrm{B} \bmod p^{m}$ we construct the solution-sets $\bmod p^{m+1}$ by means of the criteria of Theorem 1. 


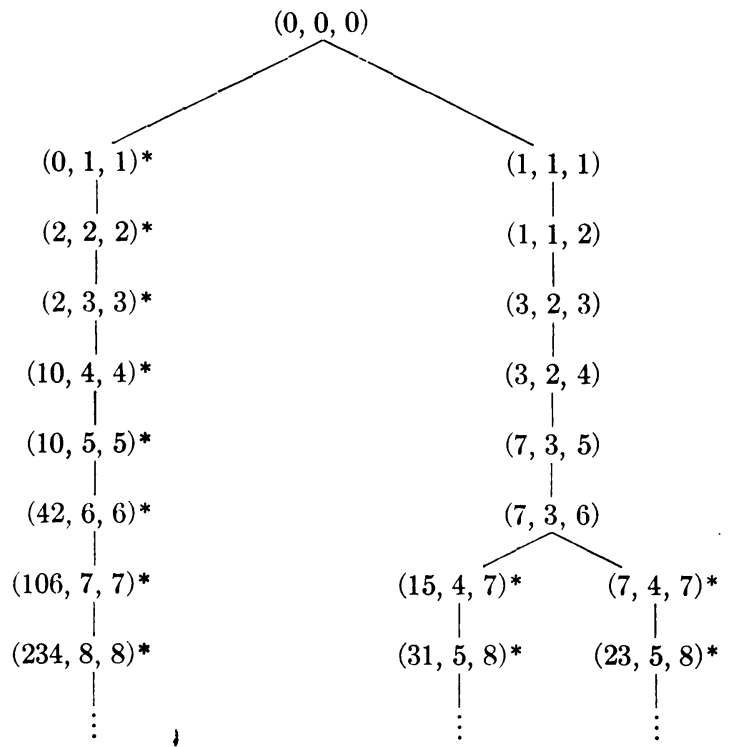

FIg. 1. Solution-sets of $(x+1)\left(x^{2}-x+6\right)=0\left(\bmod 2^{m}\right)$. The solution-sets of type A are indicated by $*$.

5. Interpretation in the $p$-adic Field. The solutions of $f(x) \equiv 0$ to arbitrary high powers of $p$ correspond to the solution of $f(x)=0$ in the $p$-adic field. In this interpretation a solution-set $(x, b, m)$ corresponds to an interval in which $f(x)$ is small in the $p$-adic valuation; specifically, $|f(y)|_{p} \leqq p^{-m}$ for $|y-x|_{p} \leqq p^{-b}$. The relevance of the definition of type of solution-sets is indicated by Theorem 1 . If $(x, b, m)$ is a solution-set of type A then, by induction of Theorem 1 (i), there is a unique solution $y$ of $f(y)=0$ in $|y-x|_{p} \leqq p^{-b}$. On the other hand, if $(x, b, m)$ is a solution-set of type B then although $|f(y)|_{p}$ is "small" in the range $|y-x|_{p} \leqq p^{-b}$ there may be no solutions of $f(y)=0$ in this range, or one or more solutions. Theorem 2 exhibits the operation of the Newton-Raphson algorithm. The computation of $-f(x) / f^{\prime}(x)$ corresponds to solving equation (7) to modulus $p^{\infty}$. For computational purposes we must be satisfied with solving the equation to modulus some suitably high power of $p$. Restriction of the algorithm to solution-sets of type A both guarantees that the iteration converges (in the $p$-adic topology) and indicates the "right" modulus in which to solve equation (7), namely $p^{2 m-2 l}$. By "right" we mean that no greater modulus will guarantee a smaller value of $\left|f\left(x^{\prime}\right)\right|_{p}$ for the next iterate $x^{\prime}$.

From the $p$-adic interpretation it also follows that there are no type B solutions for some sufficiently large modulus, unless the rational polynomial $f(x)$ has a repeated factor. For if $\left(x_{n}, b, n\right)$ is a convergent sequence of type $\mathrm{B}$ solution-sets then $\left|f\left(x_{n}\right)\right|_{p} \leqq p^{-n}$ and $\left|f^{\prime}\left(x_{n}\right)\right|_{p} \leqq p^{-l} \leqq p^{-n / 2}$. Hence $\lim _{n} x_{n}$ is a root of both $f(x)$ and $f^{\prime}(x)$. Further, the existence of a common root of $f(x)$ and $f^{\prime}(x)$ in the $p$-adic field implies a repeated factor of the rational polynomial $f(x)$ since the two discriminants are formally the same.

6. The Search for Close-Packed Codes. The existence of a close-packed errorcorrecting binary code [2] requires integers $x, r$ with 


$$
f_{r}(x) \equiv r !\left\{1+x+\left(\begin{array}{l}
x \\
2
\end{array}\right)+\cdots+\left(\begin{array}{l}
x \\
r
\end{array}\right)\right\}=2^{k} .
$$

The algorithm described in $\S 4$ was programmed for the IBM 704 to search for solutions of $f_{r}(x) \equiv 0\left(\bmod 2^{m}\right)$. For all $m, r$ with $2 \leqq r \leqq 20$ and $0 \leqq m \leqq 139$ the least value of $x$ with

$$
\begin{gathered}
0 \leqq x<2^{70}, \\
f_{r}(x) \equiv 0\left(\bmod 2^{m}\right)
\end{gathered}
$$

and

$$
f_{r}(x) \not \equiv 0\left(\bmod 2^{m+1}\right)
$$

was printed and also an indication of whether or not

$$
x<r \cdot 2^{[(m+r-1) / r]} .
$$

Finally it was determined for each value of $r$ that there were no solutions of $f_{r}(x) \equiv 0\left(\bmod 2^{140}\right)$ with $0 \leqq x<2^{70}$. Now if $f_{r}(x)=(r !) \cdot 2^{k}$ with $0 \leqq x<2^{70}$ then either $k+s \geqq 140$ (where $2^{s} \| r$ !) or equations (9) hold with $m=k+s$. In the latter case inequality (10) must also be satisfied. For if not, then $x \geqq r \cdot 2^{m / r}$ and hence $f_{r}(x) \geqq(x-r)^{r} \geqq r^{r}\left(2^{m / r}-1\right)^{r} \geqq r^{r}\left(3 \cdot 2^{m / r} / 4\right)^{r}=(3 r / 4)^{r} \cdot 2^{m}>$ $(r !) \cdot 2^{m}>(r !) \cdot 2^{k}$.

The only solutions of $(9)$ and (10) found for $2 \leqq r \leqq 20$ and $2 r+1<x$ were $x=90, r=2$ and $x=23, r=3$. Hence there are no solutions of $f_{r}(x)=(r !) \cdot 2^{k}$ for $2 \leqq r \leqq 20$ and $0 \leqq x<2^{70}$ other than

(i) $0 \leqq x \leqq r$ for arbitrary $r$; these do not correspond to close-packed codes.

(ii) $x=2 r+1$ for arbitrary $r$; these correspond to the trivial $r$ error-correcting codes of two code points of length $2 r+1$. [1].

(iii) $x=90, r=2$; this does not correspond to a close-packed code as shown in

(iv) $x=23, r=3$; this corresponds to the Golay-Paige code of $2^{12}$ code points of length $23[1,3]$.

IBM Watson Research Center Yorktown Heights, New York

1. M. J. E. Golay, "Notes on digital coding," Proc. IRE, v. 37, 1949, p. 657.

2. R. W. Hamming, "Error detecting and error correcting codes," Bell Systems Tech. J., v. 24, 1950, p. 147-160. MR 12, 35.

3. Lowell J. Paige, "A note on the Mathieu groups," Canad. J. Math. v. 9, 1957, p. 15-18. MR 18, 871.

4. J. L. Selfridge, Private Communication.

5. H. S. Shapiro \& D. L. Slotnick, "On the mathematical theory of error-correcting codes," IBM J. Res. Develop., v. 3, 1959, p. 25-34. MR 20 \$5092. 